\title{
Non-solvable contractions of semisimple Lie algebras in low dimension
}

\author{
R. Campoamor-Stursberg $\dagger$ \\ $\dagger$ Dpto. Geometría y Topología \\ Fac. CC. Matemáticas \\ Universidad Complutense de Madrid \\ Plaza de Ciencias, 3 \\ E-28040 Madrid, Spain \\ E-mail: rutwig@mat.ucm.es
}

\begin{abstract}
The problem of non-solvable contractions of Lie algebras is analyzed. By means of a stability theorem, the problem is shown to be deeply related to the embeddings among semisimple Lie algebras and the resulting branching rules for representations. With this procedure, we determine all deformations of indecomposable Lie algebras having a nontrivial Levi decomposition onto semisimple Lie algebras of dimension $n \leq 8$, and obtain the non-solvable contractions of the latter class of algebras.
\end{abstract}

PACS numbers: 02.20Sv, 02.20Qs 


\section{Introduction}

Contractions of Lie algebras have played a major role in physical applications, starting from the pioneering work of Segal and Inönü and Wigner [1, 2] up to the many generalizations of the contraction notion developed over the decades [3]. Early in the development of the theory of contractions, its relation to a somewhat inverse procedure, that of deformations of Lie algebras, was recognized and developed in [4], and tested

for consistency in the case of three dimensional algebras. An important consequence of this work was the fact that the Lie algebras contracting onto a given Lie algebra $\mathfrak{g}$ had to be searched among the deformations of the latter, thus establishing the invertibility of contractions $₫$ The introduction of further techniques like the cohomology of Lie algebras [5] allowed one to interpret contractions geometrically in the variety of Lie algebras having a fixed dimension. Once the most important groups intervening in applications were analyzed, like the Lie algebras in the classical and quantum relativistic kinematics, the attention of various authors was turned to obtain complete diagrams of contractions in low dimension [6], which have been enlarged and completed in order to cover all the special types of contractions considered earlier [7]. Such lists have been obtained up to dimension 4 over the field of real numbers. In this approach, the analysis depends essentially on a reliable classification of real Lie algebras, which only exists up to dimension six. For higher dimensions, only partial results have been obtained, and the absence of a classification of solvable non-nilpotent algebras constitutes an important obstruction in studying contractions for any fixed dimension.

In this work, we approach the contraction problem from another point of view. Instead of fixing the dimension, we focus on the structure of the contracting Lie algebras. To this extent, we choose the semisimple Lie algebras up to dimension 8, and determine the non-solvable contractions. It turns out that the Levi decomposition and the embedding problem of semisimple Lie algebras, as well as the branching rules of representations, play a prominent role in this analysis. Actually, Levi subalgebras of Lie algebras have a certain stability property that allows one to control, up to some extent, how the deformations and contractions behave [5]. Using the reversibility of contractions, we determine the deformations of low dimensional Lie algebras $\mathfrak{g}$ having a nontrivial Levi decomposition, i.e., such that they decompose into $\mathfrak{g} \vec{\oplus}_{R} \mathfrak{r}$ with $\mathfrak{s} \neq 0$ semisimple, $\mathfrak{r} \neq 0$ the radical and $R$ a nontrivial representation of the semisimple part acting by derivations on the radical. In particular, we determine which deformations lead to a semisimple Lie algebra, and obtain the corresponding contraction. For decomposable contractions, i.e., algebras decomposing as direct sum of ideals, we find that they exist whenever none of the ideals is semisimple. This will imply that reductive algebras can only appear as contractions of decomposable algebras.

Unless otherwise stated, any Lie algebra $\mathfrak{g}$ considered in this work is defined over the field $\mathbb{R}$ of real numbers. We convene that nonwritten brackets are either zero or

$\ddagger$ Later it was pointed out that not every deformation is associated to a contraction. 
obtained by antisymmetry. We also use the Einstein summation convention. Abelian Lie algebras of dimension $n$ will be denoted by the symbol $n L_{1}$.

\section{Contractions, deformations and cohomology of Lie algebras}

From the geometrical point of view, a Lie algebra $\mathfrak{g}=(V, \mu)$ is a pair formed by a vector space $V$ and a bilinear alternating (i.e., skew-symmetric) tensor $\mu: V \times V \rightarrow V$ that satisfies the Jacobi identity. For any fixed basis of $V$, the coordinates of this tensor are identified with the structure constants $C_{i j}^{k}$ of $\mathfrak{g}$. In this sense, the set of real Lie algebra laws $\mu$ over $V$ forms a variety $\mathcal{L}^{n}$ embedded in $\mathbb{R}^{\frac{n^{3}-n^{2}}{2}}$ [8]. The coordinates of a point correspond to the structure tensor of an algebra $\mathfrak{g}$. Since the general linear group acts naturally on this variety, the orbits $\mathcal{O}(\mathfrak{g})$ of a point $\mathfrak{g}$ (i.e., a Lie algebra) are formed by all Lie algebras isomorphic to $\mathfrak{g}$. Deformations of Lie algebras arise from the problem of studying the properties of these orbits. This leads one to analyze neighborhoods of a given Lie algebra in the variety, as well as the intersection of orbits corresponding to different Lie algebras. Of special interest are the so called stable Lie algebras, which are those for which the orbit $\mathcal{O}(\mathfrak{g})$ is an open set [5]. One of the main tools in this analysis is the adjoint cohomology of Lie algebras [8].

Recall that an $\mathrm{n}$-cochain $\varphi$ of a Lie algebra $\mathfrak{g}=(V, \mu=[.,]$.$) is a multilinear$ antisymmetric map $\varphi: V \times{ }^{n} \cdot \times V \rightarrow M$, where $M$ is a $\mathfrak{g}$-module. For the special case $M=V$, we get the vector space $C^{n}(V, V)$ of $n$-cochains with values in the adjoint module $\$$ By means of the coboundary operator

$$
\begin{gathered}
d \varphi\left(X_{1}, . ., X_{n+1}\right)=\sum_{i=1}^{n+1}(-1)^{i+1}\left[X_{i}, \varphi\left(X_{1}, . ., \widehat{X}_{i}, . ., X_{n+1}\right)\right]+ \\
\sum_{1 \leq i, j \leq n+1}(-1)^{i+j} \varphi\left(\left[X_{i}, X_{j}\right], X_{1}, . ., \widehat{X}_{i}, . ., \widehat{X}_{j}, . . X_{n+1}\right)
\end{gathered}
$$

we obtain a cochain complex $\left\{d: C^{n}(V, V) \rightarrow C^{n+1}(V, V), \quad n \geq 0\right\}$. In particular, $d \circ d=0$ holds. We call $\varphi \in C^{n}(V, V)$ a $n$-cocycle if $d \varphi=0$, and a $n$-coboundary if there exists $\sigma \in C^{n-1}(V, V)$ such that $d \sigma=\varphi$. The spaces of cocycles and coboundaries are denoted by $Z^{n}(V, V)$, respectively $B^{n}(V, V)$. By (1), we have the inclusion relation $B^{n}(V, V) \subset Z^{n}(V, V)$ for all $n$, and the quotient space

$$
H^{n}(V, V)=Z^{n}(V, V) / B^{n}(V, V)
$$

is called $n$-cohomology space of $\mathfrak{g}$ for the adjoint representation [9]. Among the many applications of these spaces [5, 10, 11, 12], they are relevant for the study of orbits in the following sense. A formal one-parameter deformation $\mathfrak{g}_{t}$ of a Lie algebra $\mathfrak{g}=(V,[.,]$.$) is$ given by a deformed commutator:

$$
[X, Y]_{t}:=[X, Y]+\psi_{m}(X, Y) t^{m}
$$

$\S$ By the identification of $\mathfrak{g}$ with the pair $(V, \mu)$, we can further suppose that the Lie bracket [.,.] is given by $[X, Y]=\mu(X, Y)$ for all $X, Y \in V$. 
where $t$ is a parameter and $\psi_{m}: V \times V \rightarrow V$ is a skew-symmetric bilinear map. Imposing that these formal brackets satisfy the Jacobi identity (up to quadratic order of $t$ ), one obtains the following expression:

$$
\begin{aligned}
& {\left[X_{i},\left[X_{j}, X_{k}\right]_{t}\right]_{t}+\left[X_{k},\left[X_{i}, X_{j}\right]_{t}\right]_{t}+\left[X_{j},\left[X_{k}, X_{i}\right]_{t}\right]_{t}} \\
& =t d \psi_{1}\left(X_{i}, X_{j}, X_{k}\right)+t^{2}\left(\frac{1}{2}\left[\psi_{1}, \psi_{1}\right]+d \psi_{2}\right)\left(X_{i}, X_{j}, X_{k}\right)+\mathcal{O}\left(t^{3}\right),
\end{aligned}
$$

where $d \psi_{l}$ is the trilinear map of (11) for $n=2$ and $\left[\psi_{1}, \psi_{1}\right]$ is defined by

$\frac{1}{2}\left[\psi_{1}, \psi_{1}\right]\left(X_{i}, X_{j}, X_{k}\right):=\psi_{1}\left(\psi_{1}\left(X_{i}, X_{j}\right), X_{k}\right)+\psi_{1}\left(\psi_{1}\left(X_{j}, X_{k}\right), X_{i}\right)+\psi_{1}\left(\psi_{1}\left(X_{k}, X_{i}\right), X_{j}\right)$

If equation (4) equals zero, then we have the conditions

$$
\begin{aligned}
& d \psi_{1}\left(X_{i}, X_{j}, X_{k}\right)=0, \\
& \frac{1}{2}\left[\psi_{1}, \psi_{1}\right]\left(X_{i}, X_{j}, X_{k}\right)+d \psi_{2}\left(X_{i}, X_{j}, X_{k}\right)=0 .
\end{aligned}
$$

Equation (6) shows that $\psi_{1}$ is a 2-cocycle in $H^{2}(\mathfrak{g}, \mathfrak{g})$, implying that deformations are generated by 2-cocycles $\|$. On the other hand, equation (7) implies that the deformation satisfies a so-called integrability condition. Additional conditions are obtained if the deformed bracket is developed up to higher orders of $t$ [8, 11]. In particular, if for some $\psi_{1} \in Z^{2}(\mathfrak{g}, \mathfrak{g})$ we have $\left[\psi_{1}, \psi_{1}\right]=0$, then the cocycle is called integrable and the linear deformation $\mathfrak{g}+t \psi_{1}$ defines a Lie algebra.

If the algebra $\mathfrak{g}_{t}$ is isomorphic to $\mathfrak{g}$, the deformation $\mathfrak{g}_{t}$ is called trivial. It is not difficult to show that if this happens, then we can find a non-singular map $f_{t}: V \rightarrow V$ such that $f_{t}\left([X, Y]_{t}\right)=\left[f_{t} X, f_{t} Y\right]$ for all $X, Y \in V$. This means that $\psi_{1}=d f_{t}$, and the cocycle is trivial (i.e., a coboundary). Therefore trivial deformations are generated by 2-coboundaries [10]. In this framework, contractions and deformations of Lie algebras can be related using trivial deformations [12].

Classically, a contraction is defined as follows: Let $\mathfrak{g}$ be a Lie algebra and $\Phi_{t} \in A u t(\mathfrak{g})$ a family of non-singular linear maps of $\mathfrak{g}$, where $t \in[1, \infty)$. For any $X, Y \in \mathfrak{g}$, the bracket over the transformed basis has the form

$$
[X, Y]_{\Phi_{t}}:=\Phi_{t}^{-1}\left[\Phi_{t}(X), \Phi_{t}(Y)\right]
$$

If the limit

$$
[X, Y]_{\infty}:=\lim _{t \rightarrow \infty} \Phi_{t}^{-1}\left[\Phi_{t}(X), \Phi_{t}(Y)\right]
$$

exists for any $X, Y \in \mathfrak{g}$, then equation (9) defines a Lie algebra $\mathfrak{g}^{\prime}$ called the contraction of $\mathfrak{g}$ (by $\Phi_{t}$ ), non-trivial if $\mathfrak{g}$ and $\mathfrak{g}^{\prime}$ are non-isomorphic Lie algebras. Further, it is not difficult to see that the infinitesimal version of equation (9) is generated by a coboundary [10]. In fact, if we consider a trivial cocycle $\psi \in B^{2}(\mathfrak{g}, \mathfrak{g})$, let $\sigma$ be the 1-cochain such that $d \sigma=\psi$. Using the exponential map we obtain the linear transformation $f_{t}=\exp (-t \sigma)$, and expressing the brackets over the transformed basis $\left\{f_{t}\left(X_{i}\right\}\right.$, we get

$$
[X, Y]_{t}=f_{t}^{-1}\left[f_{t}(X), f_{t}(Y)\right] .
$$

\| By this we mean that the linear term of the deformation is a cocycle. 
Therefore a contraction can be obtained by taking limits in (10). An important result states that for any contraction of Lie algebras $\mathfrak{g} \rightarrow \mathfrak{g}^{\prime}$ there is a deformation of $\mathfrak{g}^{\prime}$ that reverses it [4]. However, it should be remarked that a formal deformation is not necessarily related to a contraction [13, 14].

A special case is given when $H^{2}(\mathfrak{g}, \mathfrak{g})=0$. In this situation, the Lie algebra $\mathfrak{g}$ has no nontrivial deformations, and, in particular, cannot arise as a contraction. Such algebras are therefore stable. Although stable algebras with nonvanishing cohomology exist [8], this condition implies the stability of important classes of Lie algebras, such as semisimple and parabolic Lie algebras [15].

\section{Contractions and cohomology}

By the preceding results, contractions of Lie algebras can be analyzed using cohomological tools. More specifically, the deformations of Lie algebras are computed, and those being invertible provide contractions [12, 16].

In general, the effective computation of the cohomology of Lie algebras is a difficult task. However, for the case of Lie algebras having a non-trivial Levi decomposition, there exists a useful reduction, called the Hochschild-Serre spectral sequence [9]. If $\mathfrak{g}$ has the Levi decomposition $\mathfrak{g}=\mathfrak{s} \vec{\oplus}_{R} \mathfrak{r}$, where $\mathfrak{s}$ denotes the Levi subalgebra, $\mathfrak{r}$ the radical of $\mathfrak{g}$ and $R$ a representation of $\mathfrak{s}$ that acts by derivations on the radical [17], then the adjoint cohomology $H^{p}(\mathfrak{g}, \mathfrak{g})$ admits the following decomposition:

$$
H^{p}(\mathfrak{g}, \mathfrak{g}) \simeq \sum_{i+j=p} H^{i}(\mathfrak{g}, \mathbb{R}) \otimes H^{j}(\mathfrak{r}, \mathfrak{g})^{\mathfrak{g}}
$$

where $H^{j}(\mathfrak{r}, \mathfrak{g})^{\mathfrak{g}}$ is the space of $\mathfrak{g}$-invariant cocycles. These are the multilinear skewsymmetric maps $\varphi \in C^{j}(\mathfrak{r}, \mathfrak{s})$ that satisfy the coboundary operator (1) and such that

$$
\begin{gathered}
(X \varphi)\left(Y_{1}, . ., Y_{j}\right)=\left[X, \varphi\left(Y_{1}, . ., Y_{j}\right)\right]-\sum_{i=1}^{j} \varphi\left(\left[X, Y_{i}\right], Y_{1}, . \widehat{Y}_{i}, . ., Y_{j}\right)=0 \\
\forall X \in \mathfrak{s}, Y_{1}, . ., Y_{j} \in \mathfrak{r} .
\end{gathered}
$$

For the particular case $p=2$ the formula simplifies to

$$
H^{2}(\mathfrak{g}, \mathfrak{g}) \simeq H^{2}(\mathfrak{r}, \mathfrak{g})^{\mathfrak{s}}
$$

This result suggests that Levi subalgebras are stable in some sense, and that deformations are determined by appropriate modification of the brackets in the radical. This idea actually constitutes an important theorem that will be used later.

Proposition 1 Let $\mathfrak{g}=\mathfrak{s} \oplus \mathfrak{r}$ be the direct sum of a semisimple Lie algebra $\mathfrak{s}$ and an arbitrary algebra $\mathfrak{r}$. Then $H^{2}(\mathfrak{g}, \mathfrak{g}) \simeq H^{2}(\mathfrak{r}, \mathfrak{r})$.

Proof. By the Hochschild-Serre spectral sequence, formula (12) holds. As an $\mathfrak{r}$-module, the space $H^{2}(\mathfrak{r}, \mathfrak{g})^{\mathfrak{g}}$ is trivial [9], and this implies that

$$
H^{2}(\mathfrak{r}, \mathfrak{g})^{\mathfrak{s}} \simeq H^{2}(\mathfrak{r}, \mathfrak{g})^{\mathfrak{g}}
$$


It suffices therefore to consider the $\mathfrak{s}$-invariance. Now, for any $\varphi \in H^{2}(\mathfrak{r}, \mathfrak{g})^{\mathfrak{s}}$ and $X \in \mathfrak{s}, Y, Z \in \mathfrak{r}$ we have

$$
(X \varphi)(Y, Z)=[X, \varphi(Y, Z)]-\varphi([X, Y], Z)-\varphi(Y,[X, Z])=[X, \varphi(Y, Z)]=0 .
$$

because the sum is direct. Now $\varphi(Y, Z) \in \mathfrak{g}$, and by the decomposition of $\mathfrak{g}$ we can rewrite it as

$$
\varphi(Y, Z)=W_{1}+W_{2}, W_{1} \in \mathfrak{s}, W_{2} \in \mathfrak{r} .
$$

Since $\mathfrak{s}$ is semisimple, for any $X$ there exists $X^{\prime} \in \mathfrak{s}$ such that $\left[X, X^{\prime}\right] \neq 0$. By the invariance condition (13) we must have $W_{1}=0$, thus $\varphi(Y, Z) \in \mathfrak{r}$ for all $Y, Z \in \mathfrak{r}$. This proves that any invariant cochain is actually a 2-cochain of the radical, from which the assertion follows by imposing the coboundary condition.

Corollary 1 Let $\mathfrak{g}$ be an indecomposable Lie algebra with non-trivial Levi subalgebra $\mathfrak{s}$. Then $\mathfrak{g}$ cannot contract onto a direct sum $\mathfrak{s}^{\prime} \oplus \mathfrak{r}$ of a semisimple Lie algebra $\mathfrak{s}^{\prime}$ with an arbitrary Lie algebra $\mathfrak{r}$.

A direct consequence of this property is that deformations of reductive Lie algebras $\mathfrak{s} \oplus n L_{1}$ are always decomposable. In particular, they cannot appear as contractions of indecomposable Lie algebras having a nontrivial Levi decomposition or semisimple Lie algebras. Moreover, any Lie algebra $\mathfrak{s} \oplus \mathfrak{t}$ with $\mathfrak{t}$ an arbitrary $n$-dimensional algebra, contracts onto the reductive algebra $\mathfrak{s} \oplus n L_{1}$. This result does obviously not exclude the possibility that an indecomposable Lie algebra contracts onto a nonsolvable decomposable algebra, it merely states that none of the ideals intervening in the decomposition can be semisimple. Large classes of Lie algebras having this type of contractions exist, like semidirect products of semisimple and Heisenberg Lie algebras [18].

\section{Contractions of semisimple Lie algebras}

The previous interpretation of Lie algebras as points of a variety provides us with some useful criteria to study deformations and contractions. In [5], an important result concerning the topology of orbits was obtained. It makes precise the intuitive idea about stability of Levi subalgebras observed previously.

Theorem 1 [5] Let $L=(V, \mu)$ be a Lie algebra, $\mathfrak{s}$ a semisimple subalgebra of $L$ and $\mathfrak{r}$ the complementary subspace of $\mathfrak{s}$ in $V$. There exists a neighborhood $U^{\mu} \in \mathcal{L}^{n}$ of $\mu$ such that if $\mu_{1} \in U^{\mu}$, then the algebra $L_{1}=\left(V, \mu_{1}\right)$ is isomorphic to a Lie algebra $L^{\prime}=\left(V, \mu^{\prime}\right)$ that satisfies the conditions

(i) $\mu\left(X, X^{\prime}\right)=\mu^{\prime}\left(X, X^{\prime}\right), \forall X, X^{\prime} \in \mathfrak{s}$,

(ii) $\mu(X, Y)=\mu^{\prime}(X, Y), \forall X \in \mathfrak{s}, Y \in \mathfrak{r}$. 
In essence, this stability theorem, due to Page and Richardson [5], establishes that if the Lie algebra $\mathfrak{g}$ has a semisimple subalgebra $\mathfrak{s}$, then its deformations will have some subalgebra isomorphic to $\mathfrak{s}$, and that the action of $\mathfrak{s}$ on the remaining generators is preserved. Combined with the Hochschild-Serre spectral sequence, this result tells that the main information about deformations of semidirect products is codified in the radical of the algebra. As application of this theorem, we can establish the following result for non-solvable contractions of semisimple Lie algebras:

Proposition 2 Let $\mathfrak{g}=\mathfrak{s} \vec{\oplus}_{R} \mathfrak{r}$ be a contraction of a semisimple Lie algebra $\mathfrak{s}^{\prime}$. Then the following holds:

(i) there exists some semisimple subalgebra $\mathfrak{s}_{1}$ of $\mathfrak{s}^{\prime}$ isomorphic to $\mathfrak{s}$,

(ii) identifying $\mathfrak{s}$ with $\mathfrak{s}_{1}$ via an isomorphism, the adjoint representation of $\mathfrak{s}^{\prime}$ decomposes as $\left.\operatorname{ad}\left(\mathfrak{s}^{\prime}\right)\right|_{\mathfrak{s}}=\operatorname{ad}(\mathfrak{s}) \oplus R$ with respect to the embedding $\mathfrak{s} \hookrightarrow \mathfrak{s}^{\prime}$.

(iii) $\mathfrak{g}$ has at least $\operatorname{rank}\left(\mathfrak{s}^{\prime}\right)$ independent Casimir operators.

The proof is nothing but a slight variation of the stability theorem. If $\mathfrak{g}=\mathfrak{s} \vec{\oplus}_{R} \mathfrak{r}$ is a contraction of $\mathfrak{s}^{\prime}$, then there exists some deformation of $\mathfrak{g}$ reversing the contraction [13. By the stability theorem, this deformation has some subalgebra that is isomorphic to the Levi part of $\mathfrak{g}$, and acts the same way on the generators of the radical. Therefore the embedding of semisimple Lie algebras $\mathfrak{s} \hookrightarrow \mathfrak{s}^{\prime}$ induces a branching rule for representations, and the quotient algebra $\mathfrak{s}^{\prime} / \mathfrak{s}$, seen as an $\mathfrak{s}$-module, is isomorphic to the representation $R$, that is, $\left.\operatorname{ad}\left(\mathfrak{s}^{\prime}\right)\right|_{\mathfrak{s}}=\operatorname{ad}(\mathfrak{s}) \oplus R$. This proves (i) and (ii). Finally, the third condition follows from the properties of contractions of invariants [16].

Corollary 2 Let $\mathfrak{s}$ be a semisimple Lie algebra of a semisimple algebra $\mathfrak{s}^{\prime}$, and $R$ be a representation of $\mathfrak{s}$. If $\left.\operatorname{ad}\left(\mathfrak{s}^{\prime}\right)\right|_{\mathfrak{s}} \neq \operatorname{ad}(\mathfrak{s}) \oplus R$, then no Lie algebra with Levi decomposition $\mathfrak{s} \vec{\oplus}_{R} \mathfrak{r}$ ( $\mathfrak{r}$ solvable) can arise as a contraction of $\mathfrak{s}^{\prime}$.

The problem of analyzing the non-solvable contractions of semisimple Lie algebras $\mathfrak{s}^{\prime}$ is therefore reduced to analyze the deformations of Lie algebras having Levi decomposition $\mathfrak{s} \vec{\oplus}_{R} \mathfrak{r}$, where $\mathfrak{s}$ is some semisimple subalgebra of $\mathfrak{s}^{\prime}, R$ is obtained from the branching rules with respect to the embedding $\mathfrak{s} \hookrightarrow \mathfrak{s}^{\prime}$ and $\mathfrak{r}$ is a solvable Lie algebra. In view of the Hochschild-Serre reduction theorem, whether such a deformation onto a semisimple algebra is possible or not depends essentially on the structure of the radical $\mathfrak{r}$. In general, the following cases can appear when studying the deformations $\mathfrak{g}_{t}$ of $\mathfrak{s} \vec{\oplus}_{R} \mathfrak{r}$ :

(i) $\mathfrak{s}$ is a maximal semisimple subalgebra of $\mathfrak{s}^{\prime}$, and either $\mathfrak{g}_{t}$ is isomorphic to $\mathfrak{s}^{\prime}$ or there exists a solvable Lie algebra $\mathfrak{r}^{\prime}$ such that $\mathfrak{g}_{t} \simeq \mathfrak{s} \vec{\oplus}_{R} \mathfrak{r}^{\prime}$.

(ii) $\mathfrak{s}$ is not a maximal semisimple subalgebra of $\mathfrak{s}^{\prime}$. In this case, a deformation $\mathfrak{g}_{t}$ that is not semisimple is either isomorphic to a semidirect product $\mathfrak{s} \vec{\oplus}_{R} \mathfrak{r}^{\prime}$ with $\mathfrak{r}^{\prime}$ solvable, or there exists a semisimple subalgebra $\mathfrak{s}_{1}$ of $\mathfrak{s}^{\prime}$ and a representation $R_{1}$ of $\mathfrak{s}_{1}$ such that $\mathfrak{g}_{t} \simeq \mathfrak{s}_{1} \vec{\oplus}_{R_{1}} \mathfrak{r}^{\prime}$ for some solvable Lie algebra $\mathfrak{r}^{\prime}$. If the latter holds, then we have the chain $\mathfrak{s} \hookrightarrow \mathfrak{s}_{1} \hookrightarrow \mathfrak{s}^{\prime}$ of semisimple Lie algebras, and the branching rule $\operatorname{ad}\left(\mathfrak{s}_{1}\right) \oplus R_{1}=\operatorname{ad}(\mathfrak{s}) \oplus R$ is satisfied. 
Case (ii) is typical when we consider contractions of simple Lie algebras onto double inhomogeneous Lie algebras [19, 20, 21].

\section{Contractions of semisimple Lie algebras in low dimension}

In order to determine all nonsolvable contractions of semisimple Lie algebras, we must have a classification of indecomposable Lie algebras having a nontrivial Levi decomposition. The first such classifications in dimensions $n \leq 8$ are due to Turkowski [17]. In the following, we use the notation of this paper to label the Lie algebras. For completeness, the structure constants are given in Table A1 of the appendix. For notational purposes, we adopt the convention that the term $D_{j}$ for $j \in \frac{1}{2} \mathbb{Z}$ denotes the irreducible representation with highest weight $2 j$ of $\mathfrak{s l}(2, \mathbb{R})$, while $R_{4}^{I I}$ and $R_{5}$ are, respectively, the irreducible representations of dimension 4 and 5 of $\mathfrak{s o}(3)$.

Since the Levi decomposition of Lie algebras is trivial up to dimension four, we will obtain nontrivial results from dimension 5 onwards. In the following, we suppose that $\mathfrak{g}=\mathfrak{s} \vec{\oplus}_{R} \mathfrak{r}$ is an indecomposable Lie algebra with nontrivial Levi decomposition.

\section{1. $\operatorname{dim} \mathfrak{g}=5$}

If $\operatorname{dim} \mathfrak{g}=5$, then $\mathfrak{g}$ must be isomorphic to the special affine Lie algebra $\mathfrak{s a}(2, \mathbb{R})=$ $\mathfrak{s l}(2, \mathbb{R}) \vec{\oplus}_{D_{\frac{1}{2}}} 2 L_{1}$. Since it is the only Lie algebra in this dimension having a nontrivial Levi decomposition, and no five dimensional semisimple algebras exists, it must be stable, and does not arise as a contraction. Further, by corollary 1, any contraction of $\mathfrak{s a}(2, \mathbb{R})$ is necessarily a solvable Lie algebra.

\section{2. $\operatorname{dim} \mathfrak{g}=6$}

In dimension six, we have the simple Lorentz algebra $\mathfrak{s o}(3,1)$ and the semisimple Lie algebras $\mathfrak{s o}(4)=\mathfrak{s o}(3) \oplus \mathfrak{s o}(3), \mathfrak{s o}^{*}(4)=\mathfrak{s o}(3) \oplus \mathfrak{s l}(2, \mathbb{R})$ and $\mathfrak{s o}(2,2)=\mathfrak{s l}(2, \mathbb{R}) \oplus \mathfrak{s l}(2, \mathbb{R})$. Additionally, four indecomposable non-solvable algebras $L_{6, j}(j=1 . .4)$ with nontrivial Levi decomposition exist (see appendix). By proposition 2 , only the algebras $L_{6,1}=$ $\mathfrak{s o}(3) \vec{\oplus}_{a d} 3 L_{1}$ and $L_{6,4}=\mathfrak{s l}(2, \mathbb{R}) \vec{\oplus}_{D_{1}} 3 L_{1}$ can arise as a contraction of a semisimple algebra. Since these algebras are isomorphic to the inhomogeneous algebras $I \mathfrak{s o}(3)$ and $I \mathfrak{s l}(2, \mathbb{R})$, we recover the well known contractions [22]:

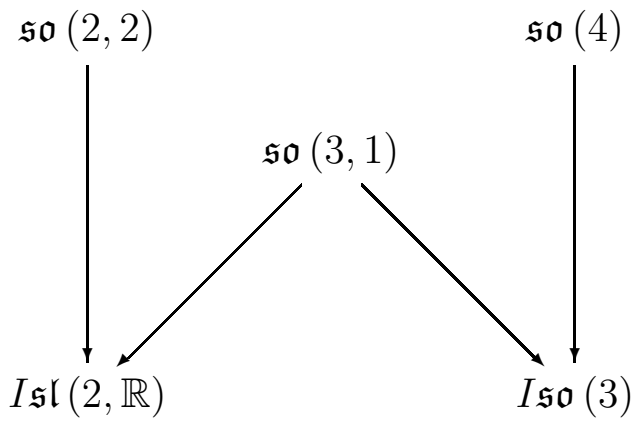


On the contrary, the semisimple algebra $\mathfrak{s o}^{*}(4)$ has no indecomposable contractions with nonzero Levi part. Any non-solvable contraction of it is necessarily a direct sum of a simple and a solvable algebra. For the remaining algebras, $L_{6,2}$ and $L_{6,3}$, it is not difficult to show, applying the Hochschild-Serre reduction theorem, that the identities $H^{2}\left(L_{6,2}, L_{6,2}\right)=H^{2}\left(L_{6,3}, L_{6,3}\right)=0$ hold, from which we deduce that these algebras are stable. It is trivial to verify that both $L_{6,2}$ and $L_{6,3}$ contract onto the decomposable algebra $\mathfrak{s a}(2, \mathbb{R}) \oplus L_{1}$. We resume the situation in the following

Proposition 3 A six dimensional indecomposable Lie algebra with nontrivial Levi decomposition is either stable or the contraction of a semisimple Lie algebra.

We remark that this result, in combination with corollary 1, provide a complete analysis of the non-solvable contractions of semisimple Lie algebras in this dimension.

\section{3. $\operatorname{dim} \mathfrak{g}=7$}

Although there are no semisimple Lie algebras in dimension seven, this dimension is of interest, since we find the lowest dimensional examples of non-solvable Lie algebras that do not arise as a contraction, but are nevertheless not stable. According to the classification in [17], there are seven isomorphism classes $L_{7, j}$, one of them depending on a continuous parameter $p$.

Proposition 4 Let $\mathfrak{g}=L_{7, j}$ with $j \neq 3$. Then $H^{2}\left(L_{7, j}, L_{7, j}\right)=0$ and $L_{7, j}$ is stable. If $\mathfrak{g}=L_{7,3}^{p}$, then

$$
\operatorname{dim} H^{2}\left(L_{7,3}^{p}, L_{7,3}^{p}\right)=\left\{\begin{array}{ll}
1, & p \neq 2 \\
2, & p=2
\end{array} .\right.
$$

Moreover, the cohomology classes are given by $\left[\varphi_{1}\right]$ and $\left[\varphi_{2}\right]$, where

$$
\varphi_{1}\left(X_{6}, X_{7}\right)=X_{6} ; \varphi_{2}\left(X_{4}, X_{5}\right)=X_{6} .
$$

The proof follows by direct computation using the Hochschild-Serre reduction. In particular, all deformations of $L_{7,3}^{p}$ for $p \neq 2$ lie in the same family, and no contraction among these algebras is possible since $\operatorname{dim} \operatorname{Der}\left(L_{7,3}^{p}\right)=8$ for all $p$, and any contraction increases the number of derivations. For $p=2$, two independent deformations are possible, since the integrability condition (77) implies that $\epsilon_{1} \epsilon_{2}=0$. The deformation $L_{7,3}^{2}+\epsilon_{1} \varphi_{1}$ is isomorphic to $L_{7,3}^{2+\epsilon_{1}}$, and clearly non-invertible by the dimension of the algebra of derivations, while the deformation $L_{7,3}^{2}+\epsilon_{2} \varphi_{2}$ has a non-abelian radical and is easily seen to be isomorphic to $L_{7,4}$. It is straightforward to verify that we obtain the contraction $L_{7,4} \longrightarrow L_{7,3}^{2}$.

Proposition 5 A seven dimensional indecomposable Lie algebra $\mathfrak{g}$ with nontrivial Levi decomposition is a contraction of a Lie algebra if and only if $\mathfrak{g} \simeq L_{7,3}^{2}$. 
In particular, the algebras of the family $L_{7,3}^{p}$ with $p \neq 2$ are neither stable nor contractions of another algebra. From this dimension onwards, this pathology appears in any dimension. We observe further that, to some extent, the stability of the remaining algebras (those not depending on a parameter) is due to the nonexistence of semisimple Lie algebras in this dimension.

\section{4. $\operatorname{dim} \mathfrak{g}=8$}

In dimension 8 , the only real semisimple Lie algebras are the real forms of $A_{2}$, that is, the compact algebra $\mathfrak{s u}(3)$ and the non-compact algebras $\mathfrak{s u}(2,1)$ and $\mathfrak{s l}(3, \mathbb{R})$. In order to obtain the contractions of these algebras that are indecomposable and have a nonzero Levi part, we must determine all possible embeddings of rank one simple subalgebras and their corresponding branching rules.

Proposition 6 Let $\mathfrak{s}^{\prime} \hookrightarrow \mathfrak{s}$ be a semisimple subalgebra of a semisimple Lie algebra $\mathfrak{s}$ of dimension 8. If the indecomposable Lie algebra $\mathfrak{g}=\mathfrak{s}^{\prime} \vec{\oplus}_{R} \mathfrak{r}$ is a contraction of $\mathfrak{s}$, then $\mathfrak{s}^{\prime}$ is a maximal simple subalgebra of $\mathfrak{s}$ and one of the following cases holds:

(i) $\mathfrak{s}^{\prime} \simeq \mathfrak{s l}(2, \mathbb{R})$ and $R=2 D_{\frac{1}{2}} \oplus D_{0}$ or $D_{1}$.

(ii) $\mathfrak{s}^{\prime} \simeq \mathfrak{s o}(3)$ and $R=R_{5}$ or $R_{4}^{I I} \oplus D_{0}$.

Proof. Since any semisimple Lie algebra in dimension 8 has rank two, a simple subalgebra is necessarily maximal as semisimple algebra. In order to obtain the admissible representations $R$, it suffices to consider the complexification $\mathfrak{g} \otimes_{\mathbb{R}} \mathbb{C}$ of $\mathfrak{g}$. Then the Levi subalgebra is isomorphic to $A_{1}$, and the problem reduces to determine the branching rule for the adjoint representation $\Gamma(1,1)_{\mathbb{C}}$ of $A_{2}$ with respect to the nonequivalent embeddings of $A_{1}$ in $A_{2}$. If the embedding $A_{1} \hookrightarrow A_{2}$ is regular [23], then the representation $\Gamma(1,1)_{\mathbb{C}}$ decomposes as

$$
\left.\Gamma(1,1)_{\mathbb{C}}\right|_{A_{1}}=D_{1} \oplus 2 D_{\frac{1}{2}} \oplus D_{0} .
$$

Since $D_{1}$ is the adjoint representation of $A_{1}$, the only possibility for $R_{\mathbb{C}}$ is $R_{\mathbb{C}}=2 D_{\frac{1}{2}} \oplus D_{0}$. Taking the real forms of $\mathfrak{s l}(2, \mathbb{C})$, we obtain that $R=2 D_{\frac{1}{2}} \oplus D_{0}$ if $\mathfrak{s}^{\prime} \simeq \mathfrak{s l}(2, \mathbb{R})$ and $R=R_{4}^{I I} \oplus D_{0}$ if $\mathfrak{s}^{\prime} \simeq \mathfrak{s o}(3)$.

For the nonregular embedding $A_{1} \hookrightarrow A_{2}$, the corresponding branching rule is easily obtained from (16), and equals

$$
\left.\Gamma(1,1)_{\mathbb{C}}\right|_{A_{1}}=\operatorname{ad}\left(A_{1}\right) \oplus R_{\mathbb{C}}=D_{2} \oplus D_{1} .
$$

Taking the real forms, we obtain the irreducible representations $D_{2}$ if $\mathfrak{s}^{\prime} \simeq \mathfrak{s l}(2, \mathbb{R})$ and $R_{5}$ if $\mathfrak{s}^{\prime}$ is compact.

In view of the classification, the only Lie algebras having the previous describing representations are $\mathfrak{F}=\left\{L_{8,2}, L_{8,3}, L_{8,4}^{p}, L_{8,5}, L_{8,13}^{\epsilon}, L_{8,14}, L_{8,15}, L_{8,16}, L_{8,17}^{p}, L_{8,18}^{p}, L_{8,21}\right\}$.

Lemma 1 The Lie algebras $L_{8,3}, L_{8,4}^{p \neq 0}, L_{8,16}, L_{8,17}^{p \neq-1}, L_{8,18}^{p \neq 0}$ do not arise as a contraction of a semisimple Lie algebra. 
Table 1. Adjoint cohomology of Lie algebras in $\mathfrak{F}$.

\begin{tabular}{|c|c|c|}
\hline$L_{8,2}$ & 1 & $\begin{array}{l}\varphi\left(X_{4}, X_{5}\right)=X_{2}, \varphi\left(X_{4}, X_{6}\right)=X_{3}, \varphi\left(X_{4}, X_{7}\right)=X_{1}, \varphi\left(X_{4}, X_{8}\right)=-\frac{3}{2} X_{5} \\
\varphi\left(X_{5}, X_{6}\right)=X_{1}, \varphi\left(X_{5}, X_{7}\right)=-X_{3}, \varphi\left(X_{5}, X_{8}\right)=\frac{3}{2} X_{4}, \varphi\left(X_{6}, X_{7}\right)=X_{2} \\
\varphi\left(X_{6}, X_{8}\right)=\frac{3}{2} X_{7}, \varphi\left(X_{7}, X_{8}\right)=-\frac{3}{2} X_{6}\end{array}$ \\
\hline$L_{8,4}^{0}$ & 2 & $\begin{array}{l}\varphi_{1}\left(X_{4}, X_{5}\right)=X_{2}, \varphi_{1}\left(X_{4}, X_{6}\right)=X_{3}+\frac{3}{2} X_{8}, \varphi_{1}\left(X_{4}, X_{7}\right)=X_{1} \\
\varphi_{1}\left(X_{5}, X_{6}\right)=X_{1}, \varphi_{1}\left(X_{5}, X_{7}\right)=-X_{3}+\frac{3}{2} X_{8}, \varphi_{1}\left(X_{6}, X_{7}\right)=X_{2} \\
\varphi_{2}\left(X_{4}, X_{8}\right)=X_{4}, \varphi_{2}\left(X_{5}, X_{8}\right)=X_{5}, \varphi_{2}\left(X_{6}, X_{8}\right)=X_{6}, \varphi_{2}\left(X_{7}, X_{8}\right)=X_{7}\end{array}$ \\
\hline$L_{8,5}$ & 1 & $\begin{array}{l}\varphi\left(X_{4}, X_{5}\right)=X_{3}, \varphi\left(X_{4}, X_{6}\right)=X_{2}, \varphi\left(X_{4}, X_{7}\right)=X_{1}, \varphi\left(X_{5}, X_{6}\right)=-X_{1} \\
\varphi\left(X_{5}, X_{7}\right)=X_{2}, \varphi\left(X_{6}, X_{7}\right)=2 X_{3}, \varphi\left(X_{6}, X_{8}\right)=-6 X_{1}, \varphi\left(X_{7}, X_{8}\right)=-6 X_{2}\end{array}$ \\
\hline$L_{8,13}^{\varepsilon}$ & 1 & $\begin{array}{l}\varphi\left(X_{4}, X_{6}\right)=-2 X_{2}, \varphi\left(X_{4}, X_{7}\right)=X_{1}, \varphi\left(X_{4}, X_{8}\right)=-3 \varepsilon X_{6}, \varphi\left(X_{5}, X_{6}\right)=X_{1} \\
\varphi\left(X_{5}, X_{7}\right)=2 X_{3}, \varphi\left(X_{5}, X_{8}\right)=-3 \varepsilon X_{7}, \varphi\left(X_{6}, X_{8}\right)=3 X_{4}, \varphi\left(X_{7}, X_{8}\right)=3 X_{5}\end{array}$ \\
\hline$L_{8,14}$ & 3 & $\begin{array}{l}\varphi_{1}\left(X_{4}, X_{8}\right)=X_{4}, \varphi_{1}\left(X_{5}, X_{8}\right)=X_{5} ; \varphi_{2}\left(X_{4}, X_{8}\right)=X_{6}, \varphi_{2}\left(X_{5}, X_{8}\right)=X_{7} \\
\varphi_{3}\left(X_{6}, X_{7}\right)=X_{8}\end{array}$ \\
\hline$L_{8,15}$ & 1 & $\begin{array}{l}\varphi\left(X_{4}, X_{5}\right)=3 X_{8}, \varphi\left(X_{4}, X_{6}\right)=-2 X_{2}, \varphi\left(X_{4}, X_{7}\right)=X_{1}, \varphi\left(X_{4}, X_{8}\right)=-3 X_{6} \\
\varphi\left(X_{5}, X_{6}\right)=X_{1}, \varphi\left(X_{5}, X_{7}\right)=2 X_{3}, \varphi\left(X_{5}, X_{8}\right)=-3 X_{7}\end{array}$ \\
\hline$L_{8,17}^{-1}$ & 2 & $\begin{array}{l}\varphi_{1}\left(X_{4}, X_{6}\right)=-2 X_{2}, \varphi_{1}\left(X_{4}, X_{7}\right)=X_{1}-3 X_{8}, \varphi_{1}\left(X_{5}, X_{6}\right)=X_{1}+3 X_{8} \\
\varphi_{1}\left(X_{5}, X_{7}\right)=2 X_{3} ; \varphi_{2}\left(X_{6}, X_{8}\right)=X_{6}, \varphi_{2}\left(X_{7}, X_{8}\right)=X_{7}\end{array}$ \\
\hline$L_{8,18}^{0}$ & 2 & $\begin{array}{l}\varphi_{1}\left(X_{4}, X_{5}\right)=3 X_{8}, \varphi_{1}\left(X_{4}, X_{6}\right)=-2 X_{2}, \varphi_{1}\left(X_{4}, X_{7}\right)=X_{1}, \varphi_{1}\left(X_{5}, X_{6}\right)=X_{1}, \\
\varphi_{1}\left(X_{5}, X_{7}\right)=2 X_{3}, \varphi_{1}\left(X_{6}, X_{7}\right)=3 X_{8} . \\
\varphi_{2}\left(X_{6}, X_{8}\right)=X_{6}, \varphi_{2}\left(X_{7}, X_{8}\right)=X_{7} .\end{array}$ \\
\hline$L_{8,21}$ & 1 & $\begin{array}{l}\varphi\left(X_{4}, X_{7}\right)=-2 X_{2}, \varphi\left(X_{4}, X_{8}\right)=X_{1}, \varphi\left(X_{5}, X_{6}\right)=6 X_{2}, \varphi\left(X_{5}, X_{7}\right)=-2 X_{1} \\
\varphi\left(X_{5}, X_{8}\right)=2 X_{3}, \varphi\left(X_{6}, X_{7}\right)=-6 X_{3}\end{array}$ \\
\hline
\end{tabular}

The proof follows at once observing that these algebras satisfy the condition $\mathcal{N}(\mathfrak{g})=0$, where $\mathcal{N}(\mathfrak{g})$ denotes the number of independent invariants for the coadjoint representation. By proposition 2, they cannot be contractions of a Lie algebra having invariants. For the remaining algebras, the existence or not of contractions cannot be deduced from the usual numerical invariants that are preserved or increased by contraction In order to analyze whether they are contractions of semisimple Lie algebras, we determine if they admit deformations onto semisimple algebras. To this extent, we apply the Hochschild-Serre reduction to compute a basis of $H^{2}(\mathfrak{g}, \mathfrak{g})$ and analyze the deformed bracket (3). The bases for the adjoint cohomology are given in Table 1.

Theorem 2 The indecomposable Lie algebras $L_{8,2}, L_{8,4}^{0}, L_{8,5}, L_{8,13}^{\epsilon}, L_{8,14}, L_{8,15}, L_{8,17}^{-1}, L_{8,18}^{0}$ and $L_{8,21}$ are all obtained as contractions of simple Lie algebras. More precisely,

(i) $\mathfrak{s u ( 3 )}$ contracts onto the algebras $L_{8,2}, L_{8,4}^{0}$ and $L_{8,5}$.

(ii) $\mathfrak{s u}(2,1)$ contracts onto the algebras $L_{8,2}, L_{8,4}^{0}, L_{8,13}^{1}, L_{8,14}, L_{8,15}, L_{8,18}^{0}$ and $L_{8,21}$.

(iii) $\mathfrak{s l}(3, \mathbb{R})$ contracts onto the algebras $L_{8,5}, L_{8,13}^{-1}, L_{8,14}, L_{8,15}, L_{8,17}^{-1}$ and $L_{8,21}$.

ๆ For a list of such invariants, see e.g. [7, 16]. 
Proof. We prove the assertion by direct analysis of the deformations of the preceding algebras.

(i) Let $L_{8,2}(\epsilon)=L_{8,2}+\epsilon \varphi$ be a linear deformation of $L_{8,2}$. For any value of $\epsilon$ the deformed commutator satisfies the Jacobi identity, thus defines a Lie algebra. Computing the Killing metric tensor over the basis $\left\{X_{1}, . ., X_{8}\right\}$, we obtain the matrix

$$
\kappa=\left(\begin{array}{cccccccc}
-3 & 0 & 0 & 0 & 0 & 0 & 0 & 0 \\
0 & -2 & 0 & 0 & 0 & 0 & 0 & \frac{3}{2} \epsilon \\
0 & 0 & -3 & 0 & 0 & 0 & 0 & 0 \\
0 & 0 & 0 & -6 \epsilon & 0 & 0 & 0 & 0 \\
0 & 0 & 0 & 0 & -6 \epsilon & 0 & 0 & 0 \\
0 & 0 & 0 & 0 & 0 & -6 \epsilon & 0 & 0 \\
0 & 0 & 0 & 0 & 0 & 0 & -6 \epsilon & 0 \\
0 & \frac{3}{2} \epsilon & 0 & 0 & 0 & 0 & 0 & -9 \epsilon^{2}
\end{array}\right)
$$

We have $\operatorname{det}(\kappa)=2^{2} 3^{8} 7 \epsilon^{6} \neq 0$ for $\epsilon \neq 0$, and therefore the deformation is semisimple. To identify to which real form $\mathfrak{g}_{\epsilon}$ is isomorphic, we compute the spectrum of $\kappa$ and obtain

$$
\operatorname{Spec}(\kappa)=\left\{-3,3,(-6 \epsilon)^{3},-\frac{9}{2} \epsilon^{2}-1 \pm \frac{1}{2} \sqrt{\left(9 \epsilon^{2}-\frac{3}{2}\right)+\frac{7}{4}}\right\}
$$

Since $9 \epsilon^{2}+2>\sqrt{\left(9 \epsilon^{2}-\frac{3}{2}\right)+\frac{7}{4}}$ for any $\epsilon$, the two last roots of $\operatorname{Spec}(\kappa)$ are always negative, and the signature $\sigma$ of $\kappa$ is given by

$$
\sigma(\kappa)=\left\{\begin{array}{rr}
-8, & \varepsilon>0 \\
0, & \varepsilon<0
\end{array}\right.
$$

For $\sigma=-8$ we obtain the compact Lie algebra $\mathfrak{s u}(3)$, while for $\sigma=0$ we get the pseudo-unitary algebra $\mathfrak{s u}(2,1)$ [24]. Finally, starting from the deformed bracket, applying formula (9) to the family of linear maps defined by $f_{t}\left(X_{i}\right)=X_{i},(i=$ $1,2,3), f_{t}\left(X_{i}\right)=t^{-1} X_{i},(i=4, \ldots, 7)$ and $f_{t}\left(X_{8}\right)=t^{-2} X_{8}$, we obtain the contraction of $\mathfrak{s u}(2,1)$ onto $L_{8,2}$.

(ii) Let $L_{8,4}^{0}\left(\epsilon_{1}, \epsilon_{2}\right)=L_{8,4}^{0}+\epsilon_{1} \varphi_{1}+\epsilon_{2} \varphi_{2}$ be a deformation. In this case the integrability condition is $\epsilon_{1} \epsilon_{2}=0$. It is straightforward to verify that the linear deformation $L_{8,4}^{0}\left(0, \epsilon_{2}\right)=L_{8,4}^{0}+\epsilon_{2} \varphi_{2}$ has a codimension one derived ideal, and cannot be semisimple + Considering $L_{8,4}^{0}\left(\epsilon_{1}, 0\right)$ and computing the spectrum of the Killing tensor $\kappa$, we obtain

$$
\operatorname{Spec}(\kappa)=\left\{(-3)^{3},-4,\left(-6 \epsilon_{1}\right)^{4}\right\} .
$$

Thus

$$
\sigma(\kappa)=\left\{\begin{array}{rr}
-8, & \epsilon_{1}>0 \\
0, & \epsilon_{1}<0
\end{array}\right.
$$

+ Actually this deformation leads to the Lie algebra $L_{8,4}^{\epsilon_{2}}$. Since the latter algebra has no invariants, it cannot be further deformed onto a semisimple algebra. 
and we again obtain that $L_{8,4}^{0}+\epsilon_{1} \varphi_{1} \simeq \mathfrak{s u}(3)$ if $\epsilon_{1}>0$ and $L_{8,4}^{0}+\epsilon_{1} \varphi_{1} \simeq \mathfrak{s u}(2,1)$ if $\epsilon_{1}<0$. Defining on $L_{8,4}^{0}\left(\epsilon_{1}, 0\right)$ the linear maps

$$
f_{t}\left(X_{i}\right)=X_{i},(i=1,2,3,8) ; f_{t}\left(X_{i}\right)=t^{-1} X_{i},(i=4, \ldots, 7),
$$

it follows that the contraction defined by them for $t \rightarrow \infty$ is isomorphic to $L_{8,4}^{0}$, showing the invertibility of the deformations.

(iii) For $L_{8,5}(\epsilon)=L_{8,5}+\epsilon \varphi$, the spectrum of $\kappa$ is given by

$$
\operatorname{Spec}(\kappa)=\left\{(-12)^{2},-8,-4 \epsilon,-6 \epsilon,(-24 \epsilon)^{2},-72 \epsilon\right\},
$$

thus $\sigma(\kappa)=-8$ if $\epsilon>0$ and $\sigma(\kappa)=2$ if $\epsilon<0$. This proves that $L_{8,5}(\epsilon) \simeq \mathfrak{s u}(3)$ if $\epsilon>0$ and $L_{8,5}(\epsilon) \simeq \mathfrak{s l}(3, \mathbb{R})$ otherwise. The contraction reversing the deformations are defined by the transformations

$$
f_{t}\left(X_{i}\right)=X_{i},(i=1,2,3) ; f_{t}\left(X_{i}\right)=t^{-1} X_{i},(i=4, \ldots, 8) .
$$

(iv) For $L_{8,13}^{\varepsilon}$ we consider the deformations $L_{8,13}^{\varepsilon}(\mu)=L_{8,13}^{\epsilon}+\mu \varphi$, where $\epsilon= \pm 1$. For any nonzero $\mu$, we obtain

$$
\operatorname{Spec}(\kappa)=\left\{-6,6,12,(-12 \mu)^{2},(12 \mu)^{2},-36 \epsilon \mu^{2}\right\} .
$$

The signature is $\sigma(\kappa)=0$ for $\epsilon=1$ and 2 for $\epsilon=-1$, proving that $L_{8,13}^{1}(\mu)$ is isomorphic to $\mathfrak{s u}(2,1)$ and $L_{8,13}^{-1}(\mu)$ is isomorphic to $\mathfrak{s l}(3, \mathbb{R})$. In both cases, the contractions are obtained from the changes of basis in $L_{8,13}^{\epsilon}(\mu)$ defined by

$$
f_{t}\left(X_{i}\right)=X_{i},(i=1,2,3) ; f_{t}\left(X_{i}\right)=t^{-1} X_{i},(i=4, \ldots, 7) ; f_{t}\left(X_{8}\right)=t^{-2} X_{8} .
$$

(v) Let $L_{8,15}(\varepsilon)=L_{8,15}+\varepsilon \varphi$ be a formal deformation. The computation of the Killing form gives $\operatorname{det}(\kappa)=2^{14} 3^{8} \varepsilon^{5} \neq 0$ for nonzero $\varepsilon$, and the spectrum is given by

$$
\operatorname{Spec}(\kappa)=\left\{-6,6,12,(-12 \varepsilon)^{3},(12 \varepsilon)^{2}\right\},
$$

thus $\sigma(\kappa)=0$ for positive $\varepsilon$ and $\sigma(\kappa)=2$ for $\varepsilon<0$. We obtain the deformations

$$
L_{8,15}+\varepsilon \varphi \simeq\left\{\begin{array}{ll}
\mathfrak{s u}(2,1), & \varepsilon>0 \\
\mathfrak{s l}(3, \mathbb{R}), & \varepsilon<0
\end{array} .\right.
$$

The deformations are reversed considering the linear maps

$$
f_{t}\left(X_{i}\right)=X_{i},(i=1,2,3) ; f_{t}\left(X_{i}\right)=t^{-3} X_{i},(i=4,5) ; f_{t}\left(X_{i}\right)=t^{-1} X_{i},(i=6,7) ; f_{t}\left(X_{8}\right)=t^{-2} X_{8} .
$$

(vi) The deformations of $L_{8,17}^{-1}$ were already considered in [14]. Here we only note that $L_{8,17}^{-1}+\varepsilon_{1} \varphi_{1}$ is semisimple, and that the spectrum of $\kappa$ equals $\sigma(\kappa)=2$ for any nonzero values of $\varepsilon_{1}$. We therefore obtain the deformation $L_{8,17}^{-1}+\varepsilon_{1} \varphi_{1} \simeq \mathfrak{s l}(3, \mathbb{R})$, the corresponding contraction being determined by

$$
f_{t}\left(X_{i}\right)=X_{i},(i=1,2,3,8) ; f_{t}\left(X_{i}\right)=t^{-1} X_{i},(i=4, \ldots, 7) .
$$

(vii) The integrability condition for the deformation $L_{8,18}^{0}+\varepsilon_{1} \varphi_{1}+\varepsilon_{2} \varphi_{2}$ is $\varepsilon_{1} \varepsilon_{2}=0$. Since the deformation $L_{8,18}^{0}+\varepsilon_{2} \varphi_{2}$ leads to a Lie algebra having no invariants, it cannot provide any contraction of a semisimple algebra. Considering $L_{8,18}^{0}+\varepsilon_{1} \varphi_{1}$ and computing the Killing tensor, we obtain $\operatorname{det}(\kappa)=2{ }^{14} 3^{7} \varepsilon_{1}^{4} \neq 0$ and the spectrum

$$
\operatorname{Spec}(\kappa)=\left\{-6,-4,6,12,\left(-12 \varepsilon_{1}\right)^{2},\left(12 \varepsilon_{1}\right)^{2}\right\},
$$


and in any case $\sigma(\kappa)=0$, showing that $L_{8,18}^{0}+\varepsilon_{1} \varphi_{1} \simeq \mathfrak{s u}(2,1)$. To obtain the contraction, we consider on the deformations the transformations

$$
f_{t}\left(X_{i}\right)=X_{i},(i=1,2,3,8) ; f_{t}\left(X_{i}\right)=t^{-1} X_{i},(i=4, \ldots, 7) .
$$

(viii) Since the Lie algebra $L_{8,21}$ has the Levi decomposition $\mathfrak{s l}(2, \mathbb{R}) \vec{\oplus}_{D_{2}} 5 L_{1}$ and $\operatorname{dim} H^{2}\left(L_{8,21}, L_{8,21}\right) \neq 0$, it follows from [5] that $L_{8,21}$ is the contraction of a semisimple Lie algebra. Considering $L_{8,21}+\varepsilon \varphi$, the spectrum of $\kappa$ is given by

$$
\operatorname{Spec}(\kappa)=\{-24,24,48,-48 \varepsilon,-12 \varepsilon, 12 \varepsilon, 48 \varepsilon, 72 \varepsilon\}
$$

and $\sigma(\kappa)=2$ for $\varepsilon>0, \sigma(\kappa)=0$ for $\varepsilon<0$. We thus obtain that

$$
L_{8,21}+\varepsilon \varphi \simeq\left\{\begin{array}{ll}
\mathfrak{s u}(2,1), & \varepsilon<0 \\
\mathfrak{s l}(3, \mathbb{R}), & \varepsilon>0
\end{array} .\right.
$$

In both cases, the contractions follows at once from the linear maps

$$
f_{t}\left(X_{i}\right)=X_{i},(i=1,2,3) ; f_{t}\left(X_{i}\right)=t^{-1} X_{i},(i=4, \ldots, 8) .
$$

To finish the proof, we still have to see that $L_{8,14}$ is also a contraction of the non-compact simple algebras $\mathfrak{s u}(2,1)$ and $\mathfrak{s l}(3, \mathbb{R})$. The Lie algebra $L_{8,14}$ has three cocycle classes. Considering a formal deformation $L_{8,14}\left(\varepsilon_{1}, \varepsilon_{2}, \varepsilon_{3}\right)=L_{8,14}+\varepsilon_{1} \varphi_{1}+\varepsilon_{2} \varphi_{2}+\varepsilon_{3} \varphi_{3}$, we obtain the integrability conditions

$$
\varepsilon_{1} \varepsilon_{3}=\varepsilon_{2} \varepsilon_{3}=0 .
$$

A simple calculation shows that $L_{8,14}\left(\varepsilon_{1}, \varepsilon_{2}, 0\right)$ satisfies $\mathcal{N}\left(L_{8,14}\left(\varepsilon_{1}, \varepsilon_{2}, 0\right)\right)=0$ whenever $\varepsilon_{1}+\varepsilon_{2} \neq 0$, and therefore no simple algebra can be reached. If $\varepsilon_{2}=-\varepsilon_{1}$, the deformation is isomorphic to $L_{8,17}^{-1}$. Consider in $L_{8,14}\left(\varepsilon_{1},-\varepsilon_{1}, 0\right) \simeq L_{8,17}^{-1}$ the following change of basis $X_{4}^{\prime}=2 X_{4}, X_{5}^{\prime}=2 X_{5}, X_{6}^{\prime}=X_{4}+X_{6}, X_{7}^{\prime}=X_{5}+X_{7}$,

where the remaining generators are not changed. This change is easily seen to preserve the action of $\mathfrak{s l}(2, \mathbb{R})$ over the radical. The only modified brackets are

$$
\left[X_{4}^{\prime}, X_{8}^{\prime}\right]=X_{4}^{\prime},\left[X_{5}^{\prime}, X_{8}^{\prime}\right]=X_{5}^{\prime},\left[X_{6}^{\prime}, X_{8}^{\prime}\right]=X_{4}^{\prime}-X_{6}^{\prime},\left[X_{7}^{\prime}, X_{8}^{\prime}\right]=X_{5}^{\prime}-X_{7}^{\prime} .
$$

If we now define

$f_{t}\left(X_{i}^{\prime}\right)=X_{i}^{\prime}, i=1,2,3 ; f_{t}\left(X_{i}^{\prime}\right)=\frac{1}{t^{3}} X_{i}^{\prime}, i=4,5 ; f_{t}\left(X_{i}^{\prime}\right)=\frac{1}{t} X_{i}^{\prime}, i=6,7 ; f_{t}\left(X_{8}^{\prime}\right)=\frac{1}{t^{2}} X_{8}^{\prime}$,

then, over the new transformed basis $\left\{X_{i}^{\prime \prime}=f_{t}\left(X_{i}^{\prime}\right)\right\}$, the preceding brackets are expressed by

$$
\left[X_{4}^{\prime \prime}, X_{8}^{\prime \prime}\right]=\frac{1}{t^{2}} X_{4}^{\prime \prime},\left[X_{5}^{\prime \prime}, X_{8}^{\prime \prime}\right]=\frac{1}{t^{2}} X_{5}^{\prime \prime},\left[X_{6}^{\prime \prime}, X_{8}^{\prime \prime}\right]=X_{4}^{\prime \prime}-\frac{1}{t^{2}} X_{6}^{\prime \prime},\left[X_{7}^{\prime \prime}, X_{8}^{\prime \prime}\right]=X_{5}^{\prime \prime}-\frac{1}{t^{2}} X_{7}^{\prime} .
$$

For $t \rightarrow \infty$ we recover the brackets of $L_{8,14}$, which shows that the deformation $L_{8,14}\left(\varepsilon_{1},-\varepsilon_{1}, 0\right)$ is invertible. The contraction

$$
\mathfrak{s l}(3, \mathbb{R}) \longrightarrow L_{8,14}
$$

follows from transitivity of contractions [13].

Finally, if we consider the deformation $L_{8,14}\left(0,0, \varepsilon_{3}\right)$ and the change of basis $X_{8}^{\prime}=\epsilon_{3} X_{8}$, 
we immediately obtain that this deformation is isomorphic to $L_{8,15}$. The corresponding contraction of $L_{8,14}\left(0,0, \varepsilon_{3}\right)$ onto $L_{8,14}$ is given by the family of transformations

$$
f_{t}\left(X_{i}\right)=X_{i},(i=1,2,3,8) ; \quad f_{t}\left(X_{i}\right)=t^{-1} X_{i},(i=4,5,6,7) .
$$

Again, by transitivity of contractions, we obtain the previous contraction (19) and also

$$
\mathfrak{s u}(2,1) \longrightarrow L_{8,14} \text {. }
$$

It remains to obtain the contractions on decomposable Lie algebras $\mathfrak{g}=\mathfrak{g}_{1} \oplus \mathfrak{g}_{2}$ with nonzero Levi part. By corollary 1 , none of the ideals $\mathfrak{g}_{i}$ can be semisimple. We can therefore assume that $\mathfrak{g}_{1}$ has the form $\mathfrak{g}_{1}=\mathfrak{s}^{\prime} \vec{\oplus}_{R} \mathfrak{r}$, where $\mathfrak{s}^{\prime}$ is simple of rank one and $5 \leq \operatorname{dim} \mathfrak{g}_{1} \leq 7$. Then $\mathfrak{g}$ can be rewritten as

$$
\mathfrak{g}=\mathfrak{s}^{\prime} \vec{\oplus}_{R \oplus k D_{0}}\left(\mathfrak{r} \oplus \mathfrak{g}_{2}\right),
$$

where $k=1,2,3$. Further, the embedding $\mathfrak{s}^{\prime} \hookrightarrow \mathfrak{s}$ induces the branching rule

$$
\left.\operatorname{ad}(\mathfrak{s})\right|_{\mathfrak{s}^{\prime}}=\operatorname{ad}\left(\mathfrak{s}^{\prime}\right) \oplus R \oplus k D_{0} .
$$

By proposition 6, the multiplicity of the trivial representation $D_{0}$ is at most one, from which it follows at once that the only possibilities are $k=1, R=R_{4}^{I I}$ if $\mathfrak{s}^{\prime} \simeq \mathfrak{s o}(3)$ and $k=1, R=2 D_{\frac{1}{2}}$ if $\mathfrak{s}^{\prime} \simeq \mathfrak{s l}(2, \mathbb{R})$. This means that the simple algebras $\mathfrak{s u}(3), \mathfrak{s u}(2,1)$ and $\mathfrak{s l}(3, \mathbb{R})$ can only contract onto the algebras $L_{7,2} \oplus L_{1}$ and $L_{7,7} \oplus L_{1}$.

Proposition 7 The Lie algebra $L_{7,2} \oplus L_{1}$ is a contraction of $\mathfrak{s u}(3)$ and $\mathfrak{s u}(2,1)$, while $L_{7,7} \oplus L_{1}$ is a contraction of $\mathfrak{s u}(2,1)$ and $\mathfrak{s l}(3, \mathbb{R})$.

Proof. We prove the assertion for $L_{7,2} \oplus L_{1}$, the reasoning being similar for the remaining case. With some effort it can be proved that $\operatorname{dim} H^{2}\left(L_{7,2} \oplus L_{1}, L_{7,2} \oplus L_{1}\right)=7$. Considering the cocycles defined by

$\varphi_{1}\left(X_{4}, X_{5}\right)=X_{8}, \varphi_{1}\left(X_{6}, X_{7}\right)=-X_{8}$ $\varphi_{2}\left(X_{4}, X_{8}\right)=-X_{6}, \varphi_{2}\left(X_{5}, X_{8}\right)=-X_{7}, \varphi_{2}\left(X_{6}, X_{8}\right)=X_{4}, \varphi_{2}\left(X_{7}, X_{8}\right)=X_{5}$

and the corresponding linear deformations $L\left(\epsilon_{1}\right)=\left(L_{7,2} \oplus L_{1}\right)+\epsilon_{1} \varphi_{1}, L\left(\epsilon_{2}\right)=$ $\left(L_{7,2} \oplus L_{1}\right)+\epsilon_{2} \varphi_{2}$, it is not difficult to verify, using Table A1, that following isomorphisms hold:

$$
L\left(\epsilon_{1}\right) \simeq L_{8,2}, \quad L\left(\epsilon_{2}\right) \simeq L_{8,4}^{0} .
$$

Using the structure constants of Table A1, we define the changes of basis

$$
f_{1, t}\left(X_{i}\right)=X_{i},(i=1,2,3,8) ; \quad f_{1, t}\left(X_{i}\right)=t^{-1} X_{i},(i=4,5,6,7)
$$

on $L\left(\epsilon_{1}\right)$ and

$$
f_{2, t}\left(X_{i}\right)=X_{i},(i=1, \ldots, 7) ; \quad f_{2, t}\left(X_{8}\right)=t^{-1} X_{8}
$$

on $L\left(\epsilon_{2}\right)$. A simple computation shows that the brackets

$$
[X, Y]^{\prime}=\lim _{t \rightarrow \infty} f_{k, t}^{-1}\left[f_{k, t}(X), f_{k, t}(Y)\right], \quad k=1,2
$$


are exactly those of $L_{7,2} \oplus L_{1}$. Since $\mathfrak{s u}(3)$ and $\mathfrak{s u}(2,1)$ both contract onto $L_{8,2}$ and $L_{8,4}^{0}$, the result follows from transitivity of contractions.

For $L_{7,7} \oplus L_{1}$ we also find that $\operatorname{dim} H^{2}\left(L_{7,7} \oplus L_{1}, L_{7,7} \oplus L_{1}\right)=7$. Considering the nontrivial cocycles

$$
\begin{array}{llll}
\varphi_{1}\left(X_{4}, X_{5}\right)=X_{8} ; & \varphi_{2}\left(X_{6}, X_{7}\right)=X_{8} ; & \varphi_{3}\left(X_{6}, X_{8}\right)=X_{4} ; & \varphi_{3}\left(X_{7}, X_{8}\right)=X_{5} ; \\
\varphi_{4}\left(X_{4}, X_{8}\right)=X_{4} ; & \varphi_{4}\left(X_{5}, X_{8}\right)=X_{5} ; & \varphi_{5}\left(X_{6}, X_{8}\right)=X_{6} ; & \varphi_{5}\left(X_{7}, X_{8}\right)=X_{7} ; \\
\varphi_{6}\left(X_{4}, X_{8}\right)=X_{6} ; & \varphi_{6}\left(X_{5}, X_{8}\right)=X_{7} . &
\end{array}
$$

we obtain the following isomorphisms

(i) $\mathfrak{g}_{1}=\left(L_{7,7} \oplus L_{1}\right)+\varepsilon_{1} \varphi_{1}+\varepsilon_{2} \varphi_{2} \simeq L_{13}^{\varepsilon}$,

$$
\left[f_{1, t}\left(X_{i}\right)=X_{i},(i=1,2,3,8) ; f_{1, t}\left(X_{i}\right)=t^{-1} X_{i},(i=4, \ldots, 7)\right]
$$

(ii) $\mathfrak{g}_{2}=\left(L_{7,7} \oplus L_{1}\right)+\varepsilon \varphi_{3} \simeq L_{8,14}$

$$
\left[f_{2, t}\left(X_{i}\right)=X_{i},(i=1, \ldots, 7) ; f_{2, t}\left(X_{8}\right)=t^{-1} X_{8}\right]
$$

(iii) $\mathfrak{g}_{3}=\left(L_{7,7} \oplus L_{1}\right)+\varepsilon_{1} \varphi_{2}+\varepsilon_{2} \varphi_{3} \simeq L_{8,15}$,

$\left[f_{3, t}\left(X_{i}\right)=X_{i},(i=1, \ldots, 5) ; f_{3, t}\left(X_{i}\right)=t^{-1} X_{i},(i=6,7,8)\right]$

(iv) $\mathfrak{g}_{4}=\left(L_{7,7} \oplus L_{1}\right)+\varepsilon\left(\varphi_{4}-\varphi_{5}\right) \simeq L_{8,17}^{-1}$,

$$
\left[f_{4, t}\left(X_{i}\right)=X_{i},(i=1, \ldots, 7) ; f_{4, t}\left(X_{8}\right)=t^{-1} X_{8}\right]
$$

(v) $\mathfrak{g}_{5}=\left(L_{7,7} \oplus L_{1}\right)+\varepsilon\left(\varphi_{5}-\varphi_{6}\right) \simeq L_{8,18}^{0}$,

$$
\left[f_{5, t}\left(X_{i}\right)=X_{i},(i=1, \ldots, 7) ; f_{5, t}\left(X_{8}\right)=t^{-1} X_{8}\right] \text {. }
$$

The linear maps in square brackets are defined over $\mathfrak{g}_{k}(k=1, . ., 5)$, and the limit

$$
[X, Y]^{\prime}=\lim _{t \rightarrow \infty} f_{k, t}^{-1}\left[f_{k, t}(X), f_{k, t}(Y)\right], X, Y \in \mathfrak{g}_{k}, k=1, . ., 5
$$

exists for any pair of generators, thus define a contraction. It can be easily verified that $[X, Y]^{\prime}$ reproduces the brackets of $L_{7,7} \oplus L_{1}$. Again, the contractions from the simple algebras $\mathfrak{s u}(2,1)$ and $\mathfrak{s l}(3, \mathbb{R})$ follow by transitivity of contractions.

In Figure 1 we display all the non-solvable contractions of $\mathfrak{s u}(3), \mathfrak{s u}(2,1)$ and $\mathfrak{s l}(3, \mathbb{R})$ obtained in the previous results.

\section{Concluding remarks}

We have determined all the non-solvable contractions of semisimple Lie algebras up to dimension 8. Using the stability theorem of Page and Richardson, we have obtained a first reduction of the problem, and seen that the existence of contractions is determined by the Levi decomposition of the target algebras. Moreover, it has been pointed out that the embeddings of semisimple algebras in other semisimple Lie algebras and the associated branching rules are essential for the study of deformations and contractions in the non-solvable case, and show that decomposable and indecomposable algebras must be considered separately. The next natural step of our analysis is to extend it to more ample classes of target algebras, in order to determine the contractions of semisimple algebras onto solvable Lie algebras. However, this problem can, in principle, be solved 
Figure 1. Non-solvable contractions of simple Lie algebras in dimension 8.

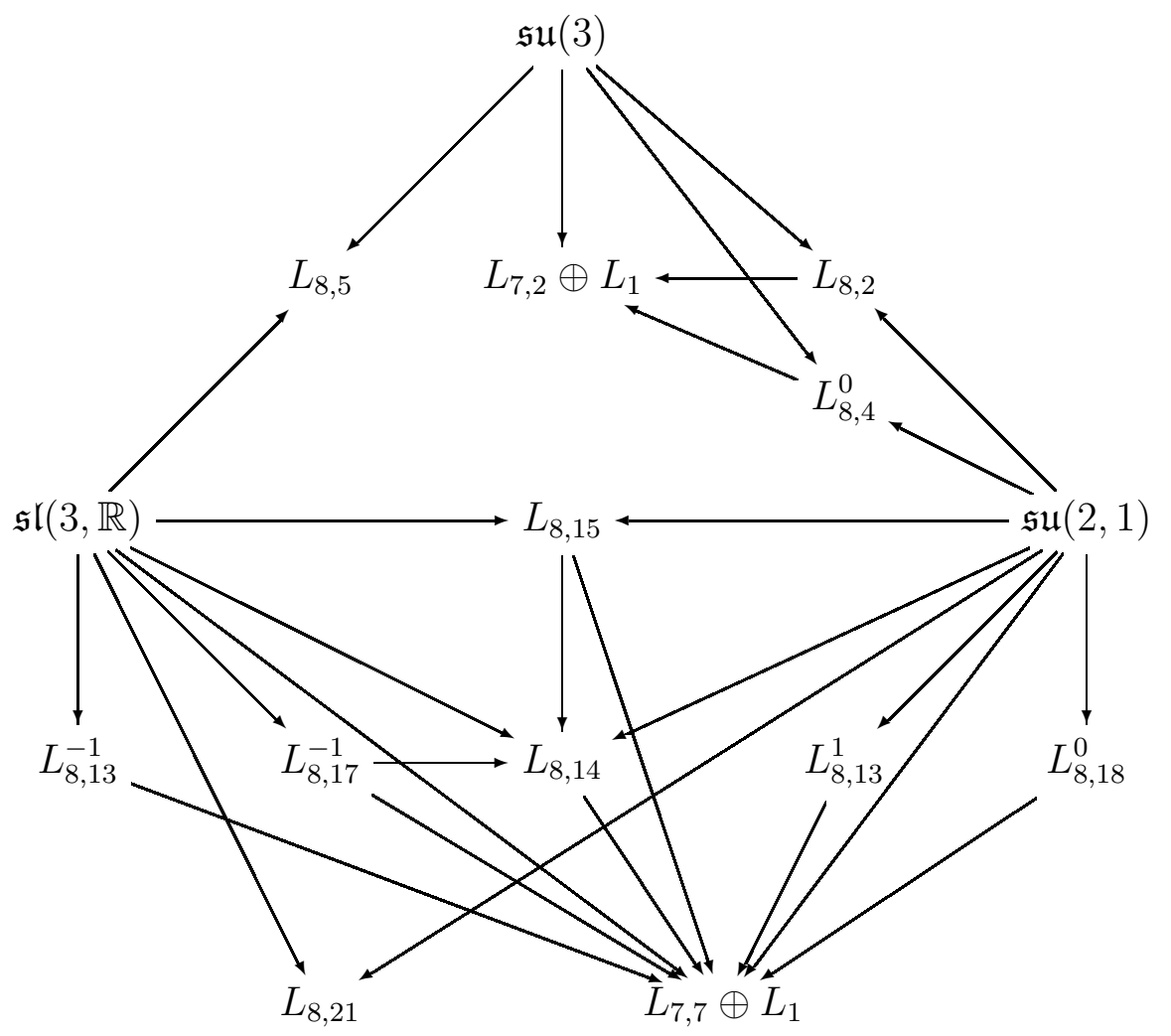

only up to dimension six, since no classification of seven dimensional solvable algebras is known. Further, the problem is technically a formidable task, not only because of the large number of isomorphism classes, but also because solvable algebras can depend on many parameters, and therefore the deformations must be analyzed for all possibilities of these parameters separately. The recent work [7] shows the difficulties that appear even in dimension four. Another possibility that is conceivable is to compute all deformations and contractions among Lie algebras with nontrivial Levi decomposition. In this sense, the only case having been analyzed corresponds to the classical kinematical algebras [25], corresponding to the representation of $\mathfrak{s o}(3)$ related to space isotropy. In the general problem, by the Page-Richardson theorem, this task is reduced to analyze the problem for Lie algebras having the same describing representation $R$. While our analysis covers the dimensions six and seven, in dimension 8 there are various parameterized families, and the exact obtainment of all possible deformations (and contractions) requires a large amount of special cases. Here the existence of many non-invertible deformations makes the analysis quite complicated. Work in this direction is actually in progress.

Among the applications of the results obtained here, we enumerate the missing 
label problem and the spontaneous symmetry breaking. Especially for the case of semisimple algebras, the knowledge of the contractions preserving some semisimple subalgebra is of interest in many situations. A special case is given by inhomogeneous Lie algebras [19, 26]. However, other types of semidirect products are relevant for many problems, such as the Galilei, Schrödinger or the Poincaré-Heisenberg algebras, and their deformations often provide additional information on the states or the configuration of a system and their invariants [12, 18, 27, 28]. In the case of the missing label problem, the contractions can be used to determine additional operators that commute with the subalgebra [18]. Finally, the obtained contractions could also be of interest in establishing relations among completely integrable systems defined on contractions of semisimple Lie algebras [29].

\section{Acknowledgements}

The author wishes to express his gratitude to the referees for useful suggestions that helped to improve the manuscript, as well as for reference [28]. This work was partially supported by the research project MTM2006-09152 of the Ministerio de Educación y Ciencia. The figures of the paper were prepared with the help of the LaTex package commutative diagrams by Paul Taylor.

\section{References}

[1] Segal I E 1951 Duke Math. J 18221

[2] Inönü E and Wigner E P 1953 Proc. Natl. Acad. Sci. U.S. 39510

[3] Saletan E 1961 J. Math. Phys. 21

Kupczyński M 1969 Comm. Math. Phys. 13154

Satyanarayana M V 1986 J. Phys. A: Math. Gen. 193697

Weimar-Woods E 1991 J. Math. Phys. 322028

[4] Levy-Nahas M 1967 J. Math. Phys. 81211

[5] Richardson R W and Page S 1967 Trans. Amer. Math. Soc. 127302

[6] Conatser C W 1972 J. Math. Phys. 13196

Huddleston P L 1978 J. Math. Phys. 191645

[7] Nesterenko I and Popovych R I 2007 J. Math. Phys. 47123515

[8] Nijenhuis A and Richardson R W 1966 Bull. Amer. Math. Soc. 721

[9] Hochschild G and Serre J-P 1953 Ann. Math. 57591

[10] Vilela Mendes R 1994 J. Phys. A: Math. Gen. 278091

[11] de Azcárraga J A and Izquierdo J M 1995 Lie Groups, Lie Algebras, Cohomology and some Applications to Physics (Cambridge: Cambridge Univ. Press)

[12] Chryssomalakos C and Okon E 2004 Int. J. Mod. Phys. D 131817

[13] Weimar-Woods E 2000 Rev. Math. Phys. 121505

[14] Campoamor-Stursberg R 2007 Phys. Lett. A to appear

[15] Tolpygo A K 1972 Mat. Zametki 42251

[16] Campoamor-Stursberg R 2003 Acta Physica Polonica B 343901

[17] Turkowski P 1988 J. Math. Phys. 292139

[18] Campoamor-Stursberg R 2005 J. Phys. A: Math. Gen. 384187

[19] Herranz F J and Santander M 1997 J. Phys. A: Math. Gen. 305411

[20] Herranz F J, Perez Bueno J C and Santander M 1998 J. Phys. A: Math. Gen. 315327 
[21] Campoamor-Stursberg R 2006 J. Phys. A: Math. Gen. 392325

[22] Weimar E 1972 Lett. Nuovo Cimento 443

[23] Onishchik A L 2003 Lectures on Real Semisimple Lie Algebras and Their Representations (Zürich: European Math. Soc.)

[24] Cornwell J F 1984 Group Theory in Physics (New York: Academic Press)

[25] Bacry H and Nuyts J 1986 J. Math. Phys. 272455

[26] Campoamor-Stursberg R 2004 Phys. Lett. A 327138

[27] Cariñena J F, Grabowski J and Marmo G 2001 J. Phys. A: Math. Gen. 343769

[28] Guillemin V and Sternberg S 1984 Symplectic Techniques in Physics (N.Y.: Cambridge Univ. Press)

Sternberg S 1975 Trans. Amer. Math. Soc. 212113

[29] Bolsinov A V 1985 Trudy Sem. Vektor. Tenzor. Anal. 228

Winternitz P 1998 Phys. Atomic Nuclei 611705

\section{Appendix}

In this appendix we give the structure constants of Lie algebras in dimension $n \leq$ 8 having a nontrivial Levi decomposition, following the notation of the original

classification [17]. The brackets are expressed by $\left[X_{i}, X_{j}\right]=C_{i j}^{k} X_{k}$ over the ordered basis $\left\{X_{1}, . ., X_{n}\right\}$ of $\mathfrak{g}$. 
Table A1. Structure constants for indecomposable Lie algebras with nontrivial Levi decomposition in dimension $n \leq 8$ after [17].

Algebra Structure constants

\begin{tabular}{|c|c|}
\hline$L_{5,1}$ & $C_{12}^{2}=2, C_{13}^{3}=-2, C_{23}^{1}=1, C_{14}^{4}=1, C_{25}^{4}=1, C_{34}^{5}=1, C_{15}^{5}=-1$ \\
\hline$\tau_{6,1}$ & $C_{23}^{1}=1, C_{12}^{3}=1, C_{13}^{2}=-1, C_{15}^{6}=1, C_{16}^{5}=-1, C_{24}^{6}=-1, C_{26}^{4}=1, C_{34}^{5}=1, C_{35}^{4}=-1$ \\
\hline$L_{6,2}$ & $C_{12}^{2}=2, C_{13}^{3}=-2, C_{23}^{1}=1, C_{14}^{4}=1, C_{25}^{4}=1, C_{34}^{5}=1, C_{15}^{5}=-1, C_{45}^{6}=1$ \\
\hline 6,3 & $C_{12}^{2}=2, C_{13}^{3}=-2, C_{23}^{1}=1, C_{14}^{4}=1, C_{25}^{4}=1, C_{34}^{5}=1, C_{15}^{5}=-1, C_{j 6}^{j}=1,(j=4,5)$ \\
\hline 6,4 & $C_{12}^{2}=2, C_{13}^{3}=-2, C_{23}^{1}=1, C_{14}^{4}=2, C_{16}^{6}=-2, C_{25}^{4}=2, C_{26}^{5}=1, C_{34}^{5}=1, C_{35}^{6}=2$ \\
\hline$L_{7,1}$ & $\begin{array}{l}C_{23}^{1}=1, C_{12}^{3}=1, C_{13}^{2}=-1, C_{15}^{6}=1, C_{16}^{5}=-1, C_{24}^{6}=-1, C_{26}^{4}=1, C_{34}^{5}=1, C_{35}^{4}=-1 \\
C_{j 7}^{j}=1(4 \leq j \leq 6)\end{array}$ \\
\hline$L_{7,2}$ & $\begin{array}{l}C_{23}^{1}=1, C_{12}^{3}=1, C_{13}^{2}=-1, C_{14}^{7}=\frac{1}{2}, C_{15}^{6}=\frac{1}{2}, C_{16}^{5}=-\frac{1}{2}, C_{17}^{4}=-\frac{1}{2}, C_{24}^{5}=\frac{1}{2} \\
C_{25}^{4}=-\frac{1}{2}, C_{26}^{7}=\frac{1}{2}, C_{27}^{6}=-\frac{1}{2}, C_{34}^{6}=\frac{1}{2}, C_{35}^{7}=-\frac{1}{2}, C_{36}^{4}=-\frac{1}{2}, C_{37}^{5}=\frac{1}{2}\end{array}$ \\
\hline$L_{7,3}$ & $\begin{array}{l}C_{12}^{2}=2, C_{13}^{3}=-2, C_{23}^{1}=1, C_{14}^{4}=1, C_{15}^{5}=-1, C_{25}^{4}=1, C_{34}^{5}=1, C_{47}^{4}=1, C_{57}^{5}=1 \\
C_{67}^{6}=p(p \neq 0)\end{array}$ \\
\hline$L_{7,4}$ & $\begin{array}{l}C_{12}^{2}=2, C_{13}^{3}=-2, C_{23}^{1}=1, C_{14}^{4}=1, C_{15}^{5}=-1, C_{25}^{4}=1, C_{34}^{5}=1, C_{45}^{6}=1, C_{47}^{4}=1 \\
C_{57}^{5}=1, C_{67}^{6}=2\end{array}$ \\
\hline$L_{7,5}$ & $\begin{array}{l}C_{12}^{2}=2, C_{13}^{3}=-2, C_{23}^{1}=1, C_{14}^{4}=2, C_{16}^{6}=-2, C_{25}^{4}=2, C_{26}^{5}=1, C_{34}^{4}=1, C_{35}^{5}=2 \\
C_{j 7}^{j}=1(j=, 4,5,6)\end{array}$ \\
\hline$L_{7,6}$ & $\begin{array}{l}C_{12}^{2}=2, C_{13}^{3}=-2, C_{23}^{1}=1, C_{14}^{4}=3, C_{15}^{5}=1, C_{16}^{6}=-1, C_{17}^{7}=-3, C_{25}^{4}=3, C_{26}^{5}=2, \\
C_{27}^{6}=1, C_{34}^{5}=1, C_{35}^{6}=2, C_{36}^{7}=3 .\end{array}$ \\
\hline$L_{7,7}$ & $\begin{array}{l}C_{12}^{2}=2, C_{13}^{3}=-2, C_{23}^{1}=1, C_{14}^{4}=1, C_{15}^{5}=-1, C_{25}^{4}=1, C_{27}^{6}=1, C_{34}^{5}=1, C_{16}^{6}=1 \\
C_{17}^{7}=-1, C_{36}^{7}=1\end{array}$ \\
\hline$L_{8,1}$ & $\begin{array}{l}C_{23}^{1}=1, C_{12}^{3}=1, C_{13}^{2}=-1, C_{15}^{6}=1, C_{16}^{5}=-1, C_{24}^{6}=-1, C_{26}^{4}=1, C_{34}^{5}=1, C_{35}^{4}=-1 \\
C_{j 8}^{j}=1(4 \leq j \leq 6), C_{78}^{7}=p\end{array}$ \\
\hline$L_{8,2}$ & $\begin{array}{l}C_{23}^{1}=1, C_{12}^{3}=1, C_{13}^{2}=-1, C_{14}^{7}=\frac{1}{2}, C_{15}^{6}=\frac{1}{2}, C_{16}^{5}=-\frac{1}{2}, C_{17}^{4}=-\frac{1}{2}, C_{24}^{5}=\frac{1}{2}, C_{25}^{4}=-\frac{1}{2}, \\
C_{26}^{7}=\frac{1}{2}, C_{27}^{6}=-\frac{1}{2}, C_{34}^{6}=\frac{1}{2}, C_{35}^{7}=-\frac{1}{2}, C_{36}^{4}=-\frac{1}{2}, C_{37}^{5}=\frac{1}{2}, C_{45}^{8}=1, C_{67}^{8}=-1 .\end{array}$ \\
\hline$L_{8,3}$ & $\begin{array}{l}C_{23}^{1}=1, C_{12}^{3}=1, C_{13}^{2}=-1, C_{14}^{7}=\frac{1}{2}, C_{15}^{6}=\frac{1}{2}, C_{16}^{5}=-\frac{1}{2}, C_{17}^{4}=-\frac{1}{2}, C_{24}^{5}=\frac{1}{2}, C_{25}^{4}=-\frac{1}{2}, \\
C_{26}^{7}=\frac{1}{2}, C_{27}^{6}=-\frac{1}{2}, C_{34}^{6}=\frac{1}{2}, C_{35}^{7}=-\frac{1}{2}, C_{36}^{4}=-\frac{1}{2}, C_{37}^{5}=\frac{1}{2}, C_{48}^{4}=1, C_{58}^{5}=1, C_{68}^{6}=1, \\
C_{78}^{7}=1\end{array}$ \\
\hline$L_{8,4}^{p}$ & $\begin{array}{l}C_{23}^{1}=1, C_{12}^{3}=1, C_{13}^{2}=-1, C_{14}^{7}=\frac{1}{2}, C_{15}^{6}=\frac{1}{2}, C_{16}^{5}=-\frac{1}{2}, C_{17}^{4}=-\frac{1}{2}, C_{24}^{5}=\frac{1}{2}, C_{25}^{4}=-\frac{1}{2}, \\
C_{26}^{7}=\frac{1}{2}, C_{27}^{6}=-\frac{1}{2}, C_{34}^{6}=\frac{1}{2}, C_{35}^{7}=-\frac{1}{2}, C_{36}^{4}=-\frac{1}{2}, C_{37}^{5}=\frac{1}{2}, C_{48}^{48}=p, C_{58}^{5}=p, C_{68}^{6}=p, \\
C_{78}^{7}=p, C_{48}^{6}=-1, C_{58}^{7}=-1, C_{68}^{4}=1, C_{78}^{5}=1 .\end{array}$ \\
\hline$L_{8,5}$ & $\begin{array}{l}C_{23}^{1}=1, C_{12}^{3}=1, C_{13}^{2}=-1, C_{14}^{7}=\frac{1}{2}, C_{15}^{6}=-\frac{1}{2}, C_{16}^{5}=2, C_{16}^{8}=-1, C_{17}^{4}=-2, C_{18}^{6}=3, \\
C_{24}^{6}=\frac{1}{2}, C_{25}^{7}=\frac{1}{2}, C_{26}^{4}=-2, C_{27}^{5}=-2, C_{27}^{8}=-1, C_{28}^{7}=3, C_{34}^{5}=2, C_{35}^{4}=-2 \\
C_{36}^{7}=1, C_{37}^{6}=-1\end{array}$ \\
\hline$L_{8,6}$ & $C_{12}^{2}=2, C_{13}^{3}=-2, C_{23}^{1}=1, C_{14}^{4}=1, C_{15}^{5}=-1, C_{25}^{4}=1, C_{34}^{5}=1, C_{45}^{8}=1, C_{67}^{8}=1$ \\
\hline$L_{8,7}^{p, q}$ & $C_{12}^{2}=2, C_{13}^{3}=-2, C_{23}^{1}=1, C_{14}^{4}=1, C_{15}^{5}=-1, C_{25}^{4}=1, C_{34}^{5}=1, C_{48}^{4}=1, C_{58}^{5}=1$ \\
\hline$p q \neq 0$ & $C_{68}^{6}=p, C_{78}^{7}=q$ \\
\hline $\begin{array}{l}L_{8,8}^{p} \\
p \neq 0\end{array}$ & $\begin{array}{l}C_{12}^{2}=2, C_{13}^{3}=-2, C_{23}^{1}=1, C_{14}^{4}=1, C_{15}^{5}=-1, C_{25}^{4}=1, C_{34}^{5}=1, C_{48}^{4}=1, C_{58}^{5}=1 \\
C_{68}^{6}=p, C_{78}^{6}=1, C_{78}^{7}=p\end{array}$ \\
\hline$L_{8.8}^{0}$ & $\begin{array}{l}C_{12}^{2}=2, C_{13}^{3}=-2, C_{23}^{1}=1, C_{14}^{4}=1, C_{15}^{5}=-1, C_{25}^{4}=1, C_{34}^{5}=1, C_{48}^{4}=1, C_{58}^{5}=1, \\
C_{78}^{6}=1\end{array}$ \\
\hline$L_{8,9}^{p, q}$ & $C_{12}^{2}=2, C_{13}^{3}=-2, C_{23}^{1}=1, C_{14}^{4}=1, C_{15}^{5}=-1, C_{25}^{4}=1, C_{34}^{5}=1, C_{48}^{4}=1, C_{58}^{5}=1$, \\
\hline$q \neq 0$ & $C_{68}^{6}=p, C_{68}^{7}=-q, C_{78}^{6}=q, C_{78}^{7}=p$ \\
\hline$L_{8,10}^{p}$ & $\begin{array}{l}C_{12}^{2}=2, C_{13}^{3}=-2, C_{23}^{1}=1, C_{14}^{4}=1, C_{15}^{5}=-1, C_{25}^{4}=1, C_{34}^{5}=1, C_{48}^{4}=1, C_{58}^{5}=1, \\
C^{6}=2 C_{7}^{7}=p C_{15}^{6}=1\end{array}$ \\
\hline
\end{tabular}


Table A2. Structure constants for indecomposable Lie algebras with nontrivial Levi decomposition in dimension $n \leq 8$ after [17] (cont.).

\begin{tabular}{|c|c|}
\hline sebra & tructure constants \\
\hline$L_{8,11}$ & $\begin{array}{l}C_{12}^{2}=2, C_{13}^{3}=-2, C_{23}^{1}=1, C_{14}^{4}=1, C_{15}^{5}=-1, C_{25}^{4}=1, C_{34}^{5}=1, C_{48}^{4}=1, C_{58}^{5}=1, \\
C_{68}^{6}=2, C_{78}^{6}=1, C_{78}^{7}=2, C_{45}^{6}=1\end{array}$ \\
\hline$L_{8,12}^{p}$ & $\begin{array}{l}C_{12}^{2}=2, C_{13}^{3}=-2, C_{23}^{1}=1, C_{14}^{4}=2, C_{16}^{6}=-2, C_{25}^{4}=2, C_{26}^{5}=1, C_{34}^{5}=1, C_{35}^{6}=2 \\
C_{48}^{4}=1, C_{58}^{5}=1, C_{68}^{6}=1, C_{78}^{7}=p\end{array}$ \\
\hline$L_{8,13}^{\varepsilon}$ & $\begin{array}{l}C_{12}^{2}=2, C_{13}^{3}=-2, C_{23}^{1}=1, C_{14}^{4}=1, C_{15}^{5}=-1, C_{25}^{4}=1, C_{34}^{5}=1, C_{16}^{6}=1, C_{17}^{7}=-1, \\
C_{27}^{6}=1, C_{36}^{7}=1, C_{45}^{8}=1, C_{67}^{8}=\varepsilon .\end{array}$ \\
\hline$L_{8,14}$ & $\begin{array}{l}C_{12}^{2}=2, C_{13}^{3}=-2, C_{23}^{1}=1, C_{14}^{4}=1, C_{15}^{5}=-1, C_{25}^{4}=1, C_{34}^{5}=1, C_{16}^{6}=1, C_{17}^{7}=-1, \\
C_{27}^{6}=1, C_{36}^{7}=1, C_{68}^{4}=1, C_{78}^{5}=1 .\end{array}$ \\
\hline$L_{8,15}$ & $\begin{array}{l}C_{12}^{2}=2, C_{13}^{3}=-2, C_{23}^{1}=1, C_{14}^{4}=1, C_{15}^{5}=-1, C_{25}^{4}=1, C_{34}^{5}=1, C_{16}^{6}=1, C_{17}^{7}=-1, \\
C_{27}^{6}=1, C_{36}^{7}=1, C_{67}^{8}=1, C_{68}^{4}=1, C_{78}^{5}=1 .\end{array}$ \\
\hline$L_{8,16}$ & $\begin{array}{l}C_{12}^{2}=2, C_{13}^{3}=-2, C_{23}^{1}=1, C_{14}^{4}=1, C_{15}^{5}=-1, C_{25}^{4}=1, C_{34}^{5}=1, C_{16}^{6}=1, C_{17}^{7}=-1, \\
C_{27}^{6}=1, C_{36}^{7}=1, C_{48}^{4}=1, C_{58}^{5}=1, C_{68}^{4}=1, C_{68}^{6}=1, C_{78}^{5}=1, C_{78}^{7}=1 .\end{array}$ \\
\hline$L_{8,17}^{p}$ & $C_{12}^{2}=2, C_{13}^{3}=-2, C_{23}^{1}=1, C_{14}^{4}=1, C_{15}^{5}=-1, C_{25}^{4}=1, C_{34}^{5}=1, C_{16}^{6}=1, C_{17}^{7}=-1$, \\
\hline $\begin{array}{l}p \neq-1 \\
L_{8,17}^{-1}\end{array}$ & $\begin{array}{l}C_{27}^{0}=1, C_{36}^{1}=1, C_{48}^{4}=1, C_{58}^{6}=1, C_{68}^{1}=p, C_{78}^{1}=p . \\
C_{12}^{2}=2, C_{13}^{3}=-2, C_{23}^{1}=1, C_{14}^{4}=1, C_{15}^{5}=-1, C_{25}^{4}=1, C_{34}^{5}=1, C_{16}^{6}=1, C_{17}^{7}=-1, \\
C_{27}^{6}=1, C_{36}^{7}=1, C_{48}^{4}=1, C_{58}^{5}=1, C_{68}^{6}=-1, C_{78}^{7}=-1\end{array}$ \\
\hline$L_{8,18}^{p}$ & $C_{12}^{2}=2, C_{13}^{3}=-2, C_{23}^{1}=1, C_{14}^{4}=1, C_{15}^{5}=-1, C_{25}^{4}=1, C_{34}^{5}=1, C_{16}^{6}=1, C_{17}^{7}=-1$, \\
\hline & $\begin{array}{l}C_{27}^{6}=1, C_{36}^{7}=1, C_{48}^{4}=p, C_{48}^{6}=-1, C_{58}^{5}=p, C_{58}^{7}=-1, C_{68}^{4}=1, C_{68}^{6}=p, C_{78}^{5}=1 \\
C_{78}^{7}=p\end{array}$ \\
\hline$L_{8,18}^{0}$ & $\begin{array}{l}C_{12}^{2}=2, C_{13}^{3}=-2, C_{23}^{1}=1, C_{14}^{4}=1, C_{15}^{5}=-1, C_{25}^{4}=1, C_{34}^{5}=1, C_{16}^{6}=1, C_{17}^{7}=-1, \\
C_{27}^{6}=1, C_{36}^{7}=1, C_{48}^{6}=-1, C_{58}^{7}=-1, C_{68}^{4}=1, C_{78}^{5}=1 .\end{array}$ \\
\hline$L_{8,19}$ & $\begin{array}{l}C_{12}^{2}=2, C_{13}^{3}=-2, C_{23}^{1}=1, C_{14}^{4}=3, C_{15}^{5}=1, C_{16}^{6}=-1, C_{17}^{7}=-3, C_{25}^{4}=3, C_{26}^{5}=2, \\
C_{27}^{6}=1, C_{34}^{5}=1, C_{35}^{6}=2, C_{36}^{7}=3, C_{47}^{8}=1, C_{56}^{8}=-3 .\end{array}$ \\
\hline$L_{8,20}$ & $\begin{array}{l}C_{12}^{2}=2, C_{13}^{3}=-2, C_{23}^{1}=1, C_{14}^{4}=3, C_{15}^{5}=1, C_{16}^{6}=-1, C_{17}^{7}=-3, C_{25}^{4}=3, C_{26}^{5}=2, \\
C_{27}^{6}=1, C_{34}^{5}=1, C_{35}^{6}=2, C_{36}^{7}=3, C_{48}^{4}=1, C_{58}^{5}=1, C_{68}^{6}=1, C_{78}^{7}=1 .\end{array}$ \\
\hline$L_{8,21}$ & $\begin{array}{l}C_{12}^{2}=2, C_{13}^{3}=-2, C_{23}^{1}=1, C_{14}^{4}=4, C_{15}^{5}=2, C_{17}^{7}=-2, C_{18}^{8}=-4, C_{25}^{4}=4, C_{26}^{5}=3, \\
C_{27}^{6}=2, C_{28}^{7}=1, C_{34}^{5}=1, C_{35}^{6}=2, C_{36}^{7}=3, C_{37}^{8}=4 .\end{array}$ \\
\hline$L_{8,22}$ & $\begin{array}{l}C_{12}^{2}=2, C_{13}^{3}=-2, C_{23}^{1}=1, C_{14}^{4}=2, C_{16}^{6}=-2, C_{17}^{7}=1, C_{18}^{8}=-1, C_{25}^{4}=2, C_{26}^{5}=1, \\
C_{28}^{7}=1, C_{34}^{5}=1, C_{35}^{6}=2, C_{37}^{8}=1 .\end{array}$ \\
\hline
\end{tabular}

